We appreciate the response to this publication feature and welcome all contributions. Contributions may be sent to José A. Mascorro, our Technical Editor, at his e-mail address: jmascor@tulane.edu. Josémay also be reached at the Department of Structural and Cellular Biology, Tulane University Health Sciences Center, 1430 Tulane Ave., New Orleans, LA 70112 and $P h:$ (504) 584-2747 (504) 584-1687

\section{Microwave Processing of Drosophila Tissues for Electron Microscopy}

\author{
JoAnn Buchanan \\ Stanford University School of Medicine \\ redhair@stanford.edu
}

Insect tissue is often difficult to prepare for electron microscopy because of the impenetrable barrier surrounding the body tissues. Drosophila salivary glands have been used for numerous studies because of the large size of the cells and their large polytene chromosomes. Early TEM studies of salivary glands (1) used a protocol that took several days. We were able to achieve excellent preservation and good ultrastructure in Drosophila salivary glands and imaginal discs from Stage L3 larvae using microwave processing in a protocol requiring less than 2 hours.

We used a Pelco Laboratory microwave (model \#3451) equipped with a Cold Spot, Steadytemp chiller/recirculator run at $15^{\circ} \mathrm{C}$, and vacuum chamber (Ted Pella, Mountain Lakes, CA) (2). The heads and attached salivary glands were removed from the animals and placed in PBS. The tissue was transferred to Pelco prep-eze specimen holders (\#36157) for ease of handling. Our goal was to use the microwave effect, not the heating effect, to prepare the tissue (3). For conventional TEM the glands and discs were prepared as follows:

1. Tissues were fixed in $2 \%$ paraformaldehyde and $2 \%$ glutaraldehyde in $0.1 \mathrm{M}$ cacodylate buffer, $\mathrm{pH} 7.4$, at power level $1(100 \mathrm{~W})$ for 1 minute on-1 minute off- 1 minute on. The power level was then changed to power level $4(450 \mathrm{~W})$ and the tissue was placed under vacuum (using house vacuum) in the chamber and pulsed for 30 seconds on-30 seconds off-30 seconds on 3 times. They were then removed from the microwave and allowed to sit for 15 minutes on the bench at room temperature.

2. Following two brief rinses in $0.1 \mathrm{M}$ cacodylate buffer containing $0.3 \mathrm{M}$ sucrose on the bench, the tissue was post-fixed in $2 \%$ osmium tetroxide containing $0.8 \%$ potassium ferricyanide for 20 seconds on- 20 seconds off- 20 seconds on at power level 1 (100 Watts) twice with cold spot at $15^{\circ} \mathrm{C}$. The tissue was removed from the microwave and allowed to sit for 15 minutes at room temperature.

3. After rinsing with distilled water, the tissue was dehydrated in an alcohol series: 50\%, 70\%, 95\%, 100 (times 3) at power level 3 (350 watts) for 30 seconds each step.

4. The tissue was infiltrated in 1 part $100 \%$ alcohol: 1 part embed 812 resin (Electron Microscopy Sciences, Fort Washington, PA) for 8 minutes at power level 3 ( 350 watts). They were changed into 2 parts resin: 1 part $100 \%$ alcohol and microwaved for $10 \mathrm{~min}$ utes at power level 3 . The tissue was placed into $100 \%$ resin and microwaved for 8 minutes at power level 3 (350 watts).

5. The discs and salivary glands were removed from the mouthparts and oriented in a flat embedding mold. The resin was hardened overnight in a $60^{\circ}$ oven.

6. Sections $1 \mu \mathrm{m}$ to $1.5 \mu \mathrm{m}$ were cut using a histoknife (Diatome) and dried flat on slides on a hotplate. Thin sections 50-70 $\mathrm{nm}$ were cut using a Diatome diamond knife and post stained

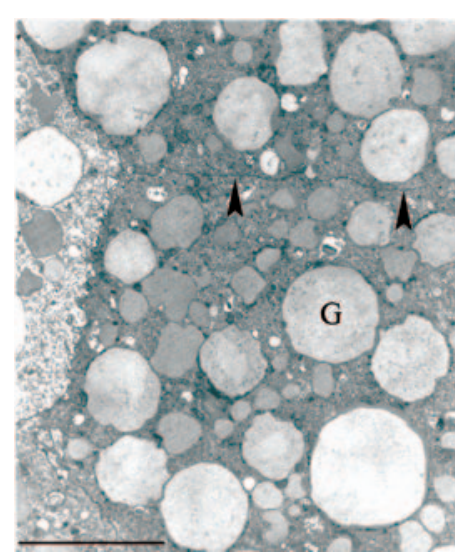

Figure 1. Drosophila salivary gland showing granules $(G)$ and septate junction (arrowheads) between the epithelial cells. Scale bar $5 \mu \mathrm{m}$

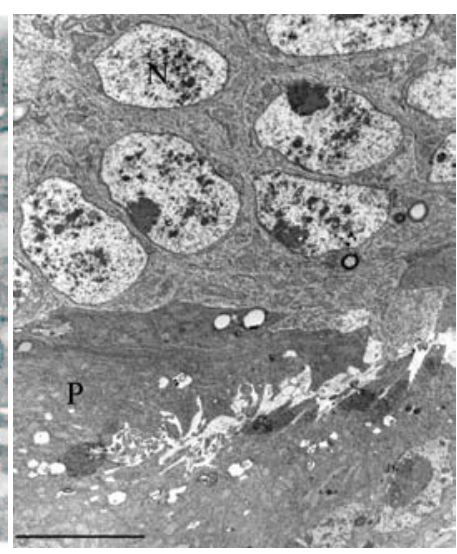

Figure 2. Drosophila wing imaginal disc showing the nuclei $(N)$ of the epithelial cells and the peripodial cell layer $(P)$. Scale bar $5 \mu \mathrm{m}$ with $5 \%$ aqueous uranyl acetate and lead citrate. Specimens were examined in a Phillips 410 Electron Microscope at $60 \mathrm{KV}$ accelerating voltage.

\section{References}

1. Lane, N.J. and Y.R. Carter. Puffs and Salivary Gland Function: The Fine Structure of the Larval and Prepupal Salivary Glands of Drosophila melanogaster. Wilhelm Roux' Archive 169:216-238, 1972.

2. Giberson, R.T. Vacuum-Assisted Microwave Processing of Animal Tissues for Electron Microscopy in Microwave Techniques and Protocols. R.T Giberson and R.S. Demaree, pp. 13-23, Humana press, Totowa, New Jersey, 2001.

3. Galvez J. J., Giberson R.T. and R.D. Cardiff. Microwave Mechanisms-The Energy/Heat Dichotomy. Microscopy Today 12:18-23.

\section{Mechanical Polishing Methods for Metal Samples for EBSD}

\author{
S. Roberts ${ }^{\star}$, D. Flatoff*, B. True Tr* $^{\star}$ \\ *South Bay Technology, Inc., San Clemente, CA 92673 \\ ${ }^{*} E D A X / T S L$, Draper, UT 84020
}

Electron backscatter diffraction is a powerful technique that has gained momentum in materials science research over the last ten years. Recent advances in electron microscope technology and automation has made EBSD a more viable and routine analytical tool [1].

Sample preparation is a key component in the use of EBSD due to the nature of the backscattered signal. Pattern formation is from the top 10-50 nm of the sample surface, and therefore, mechanical damage remaining from sample preparation will result in a poor quality signal [2]. Several methods of sample preparation have been investigated that vary in abrasive material used, preparation time, and material system. Some approaches have used a combinatorial approach of silicon carbide papers, aluminum oxide papers, and aluminum oxide suspension with a polishing cloth to prepare tantalum [3]. This protocol has yielded acceptable results, but proved to be extremely time consuming. Other methods for preparing metals, such as steels, have utilized electro-polishing with a perchloric acid electrolyte solution [4]. Electropolishing is an excellent method for preparing samples for EBSD, but can be difficult to accomplish for a wide range of materials due to the large number of acid solutions required. Recently, work has been done using focused ion beam thinning to produce samples suitable for EBSD, but problems can arise due to amorphous damage and 